\title{
MODELO EXPERIMENTAL DE ICTERÍCIA OBSTRUTIVA. AVALIAÇÃO POR MEIO DA BIOIMPEDÂNCIA
}

\author{
EXPERIMENTAL MODEL OBSTRUCTIVE JAUNDICE. ASSESSMENT BY \\ BIOIMPEDANCE
}

\author{
Flávio Daniel Saavedra Tomasich, TCBC-PR ${ }^{1}$; Adilson Alves Dias²; \\ Maria de Lourdes Pessole Biondo Simões, TCBC-PR ${ }^{3}$; Adhemar Monteiro Pacheco- TCBC-SP ${ }^{4}$; \\ Patrícia Burda Costa ${ }^{5}$, Juliana Piechnik ${ }^{6}$
}

\begin{abstract}
RESUMO: Objetivo: A bioimpedância é um método utilizado, como medida de massa corporal, volume líquido e volume de gordura corporal. Os autores analisam a utilização e desempenho da impedância em modelo de pesquisa experimental de icterícia obstrutiva. Método: Utilizaram-se oito ratos da linhagem Wistar, que foram divididos em dois grupos de quatro animais: grupo 1(experimento) e grupo 2 (controle). No grupo 1 foi realizado ligadura do colédoco e no grupo 2 laparotomia. Realizou-se a mensuração da resistência e da reatância capacitiva com aparelho de análise de bioimpedância no pré e pós-operatórios. Resultados: O estudo revelou valores médios de resistência para o grupo controle maiores do que para o experimento, mostrando uma diferença significante. Também os valores de reatância capacitiva apresentaram médias maiores no grupo controle com $\mathrm{p}<0,0001$. Conclusão: A análise dos resultados permite concluir que no rato: há diferenças nos valores de impedância entre os animais do grupo controle e experimental; os valores médios de resistência e reatância capacitiva são menores no grupo experimento e que a aplicabilidade da impedância no presente modelo pode ser sugerida (Rev. Col. Bras. Cir. 2006; 33(1): 15-18).
\end{abstract}

Descritores: Icterícia obstrutiva; Composição corporal; Modelos animais; Ratos Wistar.

\section{INTRODUÇÃO}

Há mais de um século os conceitos de impedância são descritos na literatura médica. Segundo Picollino em 1997, Luigi Galvani em 1797 foi o primeiro a publicar os conceitos de eletrofisiologia, sendo considerado o pai do método. Em 1888, Vigorous fez a primeira referência sobre impedância na literatura médica. $\mathrm{O}$ autor identificou doenças com baixa impedância como: febre, astenia, epilepsia e outras como o bócio que é uma das poucas doenças com alta impedância ${ }^{1}$.

Na década de 40 são feitos os primeiros estudos para correlacionar as medidas de impedância e a composição dos compartimentos hídricos do ser humano. Atualmente, as principais aplicações dos métodos de impedância em medicina são: a determinação de composição corporal por bioimpedância e a determinação do débito cardíaco, tanto na população adulta como na pediátrica².

A bioimpedância corporal total é um método utilizado como técnica de medidas de massa corporal, medida de volume líquido e medida de volume de gordura corporal, sendo reconhecida pelo Ministério da Saúde Brasileiro e pelo Food and Drug Administration (FDA) como valiosa técnica para tal finalidade $\mathrm{e}^{3,4,5,6,7}$.
Métodos baseados em conceitos de impedância são empregados em diversas áreas do conhecimento humano como: eletroquímica, engenharia dos materiais, eletrônica, bioengenharia, biologia e medicina, em virtude de sua facilidade de execução, precisão, praticidade, segurança e baixo custo $^{3,4,8,9,10}$.

A Conferência de Padronização sobre Análise de Impedância Bioelétrica do Instituto Nacional de Saúde Americano em 1996, considerou o método de impedância bioelétrica: seguro, sem efeitos colaterais ou contra-indicações e foi liberado sem restrições ${ }^{5}$.

O aparelho de análise de bioimpedância (BIABioimpedance Analiser) faz a leitura da resistência (R) e da reatância capacitiva $(\mathrm{Xc})$. O método tem sido útil e sensível na determinação da composição corporal e quantificação de compartimentos hídricos em diferentes condições experimentais.

O presente estudo tem como objetivo avaliar a utilização e o desempenho da impedância em modelo de pesquisa de icterícia obstrutiva em ratos; verificar se existe diferença entre os valores de resistência e reatância capacitiva obtida entre grupos; avaliar a aplicabilidade no acompanhamento da icterícia obstrutiva.

1. Professor em Técnica Cirúrgica e Cirurgia Experimental da UFPR; Médico do Hospital Erasto Gaertner-PR.

2. Professor de Fisiologia da Faculdade de Ciências Médicas da Santa Casa de São Paulo.

3. Chefe da Disciplina de Técnica Cirúrgica e Cirurgia Experimental da Universidade Federal do Paraná.

4. Professor do Departamento de Cirurgia da Faculdade de Ciências Médicas da Santa Casa de São Paulo; Chefe do Grupo de Vias Biliares.

5. Médica Residente da Cirurgia Oncológica do Hospital Erasto Gaertner.

6. Acadêmica de Medicina.

Recebido em 08/08/2005

Aceito para publicação em 10/10/2005

Conflito de interesses: nenhum

Fonte de financiamento: nenhuma

Trabalho realizado em conjunto, pela Disciplina de Técnica Cirúrgica e Cirurgia Experimental da Universidade Federal do Paraná (linha de pesquisa experimental em bioimpedância) e pela Disciplina de Cirurgia de Vias Biliares do Departamento de Cirurgia da Faculdade de Ciências Médicas da Santa Casa de São Paulo (linha de pesquisa em bioimpedância clínica). 


\section{MÉTODO}

Utilizou-se para a realização do experimento oito ratos machos (Rattus novergicus barkenhout) da linhagem Wistar, com peso médio de $300 \mathrm{~g}$ e idade de 90 dias, que foram separados de forma aleatória em dois grupos de 4 animais: experimento (grupo1) e controle (grupo 2).

Submeteu-se os animais a jejum pré-operatório de 12 horas e sob anestesia inalatória de éter etílico e em condições de assepsia, realizou-se laparotomia mediana de aproximadamente $4 \mathrm{~cm}$. Para o grupo 1 confeccionou-se ligadura do colédoco para o desenvolvimento de icterícia obstrutiva. No grupo 2 os animais foram submetidos à laparotomia seguida de laparorrafia.

Após a recuperação anestésica, os ratos permaneceram nas suas gaiolas, até o dia previsto para aferição, com livre acesso a água e ração.

A resistência (R) e a reatância capacitiva (Xc) foram aferidas com aparelho de análise de bioimpedância (BIABioimpedance Analiser) em ambos os grupos no período préoperatório e no pós-operatório imediato e diariamente até o $7^{\circ}$ pós-operatório ou o óbito do animal.

A realização da bioimpedância consiste na colocação de quatro eletrodos, sendo dois numa extremidade do corpo e dois em outra (Figura 1). Os eletrodos distais são denominados eletrodos de corrente e os eletrodos proximais são eletrodos de leitura. O aparelho de análise de bioimpedância faz a leitura da resistência e da reatância capacitiva. Esses dados são interpretados por meio de fórmulas já validadas na literatura médica e através delas, obtêm-se diretamente os dados de composição corporal.

No presente estudo, avaliou-se os valores específicos fornecidos pelo aparelho, sem aplicação de fórmulas de composição.

\section{RESULTADOS}

No grupo controle os valores de resistência variaram de 396 a 417ohms, com média de 409ohms. A reatância capacitiva do grupo controle variou de 74 a 88, com média de 82ohms.

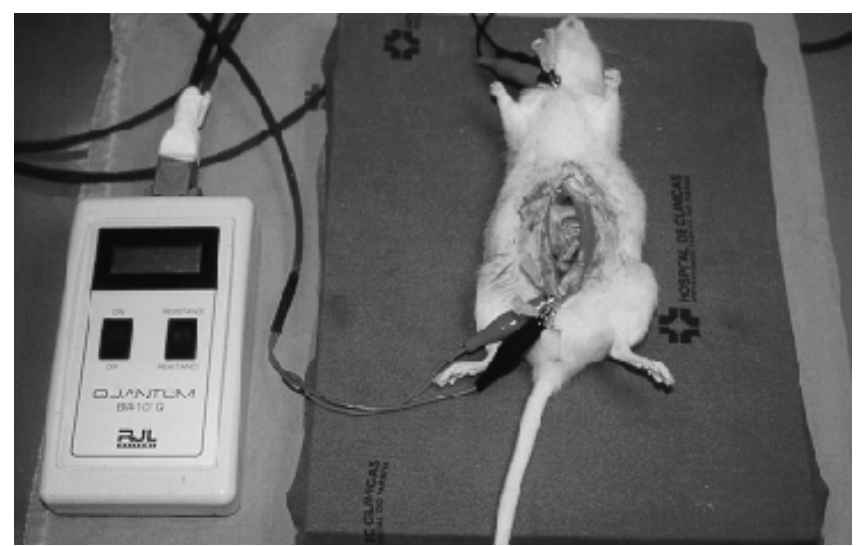

Figura 1 - Aparelho de análise de bioimpedância. Medida da resistência e reatância capacitiva.
Tabela 1 - Valores médios do Grupo 1 e 2 no dia óbito.

\begin{tabular}{lcc}
\hline & Resistência & Reatância \\
\hline Grupo Experimento & 309,5 & 66 \\
Grupo Controle & 409 & 82 \\
P & 0,001 & 0,001 \\
\hline
\end{tabular}

Nos animais do grupo experimento os valores da resistência variaram de 271 a 440ohms, com média de 365ohms. A reatância capacitiva no grupo experimento variou de 50 a 82, com média de 65ohms. Os ângulos de fase calculados por meio dos valores determinados de resistência e reatância capacitiva não foram significativos em nenhum dos grupos.

Quando comparadas às médias da resistência entre os grupos, verificou-se um $\mathrm{p}<0,0001$. Na comparação da reatância capacitiva também observou-se diferença estatística.

Comparadas as médias dos valores de resistência e reatância dos animais do grupo experimento no dia do óbito de cada um, com os valores do grupo controle, obteve-se $\mathrm{p}<0,001$, conforme a Tabela 1 .

\section{DISCUSSÃO}

Impedância é o impedimento ao livre fluxo de cargas elétricas devido a uma corrente elétrica alternada senoidal em um sistema. A impedância apresenta três componentes principais: componente resistivo - energia dissipada no sistema; componente capacitivo - energia armazenada no sistema; componente indutivo - energia gerada no sistema ${ }^{3,4,8,9}$.

Os elementos que apresentam comportamento resistivo são denominados resistores, e os elementos que apresentam a capacidade de armazenar energia são denominados de capacitores. Os capacitores são formados por duas placas paralelas de um material dielétrico (isolante) ${ }^{1}$; em termos biológicos, as membranas celulares apresentam um comportamento de capacitor sendo os condutores os meios intra e extra-celular, e o meio dielétrico a dupla camada lipídica. A base do método é a passagem de uma corrente elétrica alternada pelo organismo e a medida da resistência oferecida contra a corrente (R) e da reatância capacitiva (Xc).

Podemos expressar a impedância como coordenadas cartesianas $(\mathrm{R}, \mathrm{Xc})$ ou coordenadas polares $(\mathrm{Z}, \mathrm{q})$. No eixo das ordenadas (eixo dos $\mathrm{x}$ ), o ângulo de fase é zero; no eixo das abscissas (eixo dos Y), o ângulo de fase é de noventa graus. A distância até a origem corresponde à amplitude, denominada módulo da impedância (Z). Isto é exatamente o que faz com os números complexos representados vetorialmente, sendo que no eixo real (eixo dos $\mathrm{x}$ ), representam-se as resistências e, no eixo imaginário (eixo dos Y), as reatâncias (Figura 2$)^{8}$.

As membranas celulares apresentam uma diferença de potencial entre os seus lados intra e extracelulares geradas por um gradiente eletroquímico. Uma parcela significativa do gasto energético da célula é utilizada para manter esse gradiente por meio da bomba de $\mathrm{Na}^{+} / \mathrm{K}^{+}$e de outras estruturas ${ }^{4}$. 


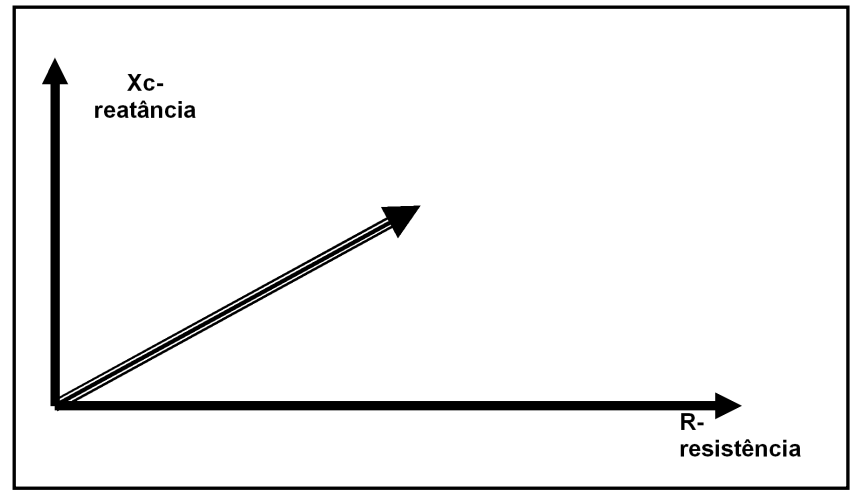

Figura 2 - Diagrama de Argand.

Como a maioria dos processos bioquímicos ocorrem na superfície da membrana ou dentro de organelas, os métodos de impedância podem ser utilizados para uma compreensão maior dos fenômenos bioelétricos e das alterações nos processos de membrana em situações de enfermidade.

O aparelho de análise de bioimpedância faz a leitura da resistência $(\mathrm{R})$ e da reatância capacitiva (Xc). Os dados da bioimpedância associados à informação do paciente como: idade, sexo, peso, altura, são aplicados em fórmulas apropriadas ou em programas de computador disponíveis comercialmente, de onde se obtêm diretamente os dados de composição corporal. Os resultados fornecidos pelos equipamentos comerciais são: água corporal total, água dos compartimentos intra e extracelular, gordura corporal total e massa magra ${ }^{3,11,12 .}$

Em condições experimentais a bioimpedância tem sido útil e sensível na determinação da composição corporal e quantificação de compartimentos hídricos. Entre as vantagens dessa técnica cita-se: método não invasivo, simples e rápido, sem contra-indicações ou efeitos colaterais, além do baixo custo $^{11}$

A partir da impedância corporal total, surge a idéia de analisar partes do corpo humano, e até de alguns tecidos, surgindo à impedância segmentar. A sua aplicação tem sido na quantificação de edema cerebral em neurocirurgia, na diferenciação entre câncer e pneumonia em massas pulmonares e na detecção de câncer de próstata em peças cirúrgicas ${ }^{11,12}$.

Neste estudo foram utilizados os conceitos de impedância na identificação de alterações físico-químicas decorrentes da icterícia provocada cirurgicamente para tentar uma correlação com uma possível complicação pós-operatória.

O estudo revelou valores médios de resistência para o grupo controle maiores do que para o grupo experimento, mostrando uma diferença estatística. Também os valores de reatância capacitiva apresentaram médias maiores no grupo controle com $\mathrm{P}<0,0001$. Os ângulos de fase avaliados pelos valores de resistência e reatância capacitiva não mostraram diferenças significativas.

Esses resultados mostram que os valores medidos pela impedância têm relação com algum evento metabólico que a icterícia obstrutiva tenha provocado nos animais do grupo experimento. A icterícia obstrutiva promove, de forma subaguda, alterações na fisiologia, justificando os valores maiores para o grupo controle do que para os animais do grupo experimental.

A análise dos resultados permite-nos concluir que: no rato há diferença nos valores de impedância obtidos entre os grupos; os valores médios de resistência e reatância capacitiva são menores no grupo experimental de icterícia obstrutiva; observou-se pior evolução no grupo experimento e, a aplicabilidade da impedância no presente modelo pode ser sugerida.

\begin{abstract}
Background: The bioimpedance is method that has been used for body mass, liquid volume and body fat volume's measurements. The authors observe the utilization and performance of the impedance in experimental research model of obstructive jaundice. Methods: Eight Wistar rats were divided in two groups with four animals: experiment (1) and control (2). In the group (1) the rats underwent a ligated choledoco bile duct surgery, and in the group (2) the rats underwent laparotomy only. The resistance and reactance measurements had been accomplished by bioelectrical impedance analysis equipment in preoperative and postoperative procedures. Results: The work denoted that the resistance's mean values for group (2) were statistically significant higher than group (1). Also, the capacitive reactance's average values were higher in the group (2) with $p<0,0001$. Conclusions: We conclude that in rats there are differences in the impedance's values between groups, the resistance and capacitive reactance's average values are lower in the experimental group.
\end{abstract}

Key words: Jaundice, obstructive; Body composition; Models, animal; Rats, Wistar.

\section{REFERÊNCIAS}

1. Piccolino M. Luigi Galvani and animal electricity: two centuries after the foundation of electrophysiology. Trends Neurosci. 1997;20(10):443-8. Erratum in: Trends Neurosci 1997;20(12):577.

2. Geddes LA, Baker LE. Principles of applied biomedical instrumentation. $3^{\text {rd }}$ ed . New York: John Wiley \& Sons; 1989.
3. Baker LE. Principles of the impedance technique. IEEE Eng Med Biol Magazine. 1989;8(1):11-5.

4. Bard AJ, Fulkner LR. Techniques based on concepts of impedance. Eletrochemical methods: fundamentals and applications. New York: John Wiley \& Sons; 1980.

5. Bioelectrical impedance analysis in body composition measurements: National Institutes of Health Office of Medical Applications of Research. Am J Clin Nutr. 1996;64(3):524S-32S. 
6. Lafortuna CL, Agosti F, Marinone PG, et al. The relationship between body composition and muscle power output in men and women with obesity. J Endocrinol Invest. 2004;27(9):85461.

7. Acuña K, Cruz T. Nutritional assessment of adults and elderly and the nutritional status of the Brazilian population. Arq Bras Endocrinol Metabol. 2004; 48(3):345-61.

8. Bonanos NL, Steele BC, Butler EP. Aplications of impedance spectroscopy. New York: John Wiley \& Sons; 1987.

9. Brett AM, Brett, CM. Métodos de impedância. Electroquímica: princípios, métodos e aplicações. Lisboa: Almedina; 1996.

10. Coppini LZ, Bottoni A, Waitzberg DL, et al. Aplicação da análise da impedância bioelétrica na avaliação nutricional. Rev Bras Nutr Clin. 1998;13(1):81-9.
11. Lee BR, Roberts WW, Smith DG, et al. Bioimpedance: novel use of a minimally invasive technique for cancer localization in the intact prostate. Prostate. 1999;39(3):213-8.

12. Gupta D, Lis CG, Dahlk SL, et al. Bioelectrical impedance phase angle as a prognostic indicator in advanced pancreatic cancer. $\mathrm{Br}$ J Nutr. 2004;92(6):957-62.

Endereço para correspondência:

Dr. Flávio Daniel Saavedra Tomasich.

Rua: Dr. Ovande do Amaral, 201. Jardim das Américas 81.520-060. Curitiba - Paraná.

E-mail: flavio@iop.com.br

Fone: 3361-5000 